\title{
Association of the 3'UTR FOXO3a Polymorphism rs4946936 with an Increased Risk of Childhood Acute Lymphoblastic Leukemia in a Chinese Population
}

\author{
Yaping Wang ${ }^{\mathrm{a}} \quad \mathrm{Li}_{\text {Zhou }}^{\mathrm{a}}$ Jing Chen ${ }^{\mathrm{b}}$ Jie Lic Lulu He ${ }^{\mathrm{a}}$ Peng Wu ${ }^{\mathrm{a}}$ Meilin Wang ${ }^{\mathrm{d}}$ \\ Na Tong ${ }^{d}$ Zhengdong Zhang ${ }^{d}$ Yongjun Fang ${ }^{a}$
}

\begin{abstract}
aDepartment of Hematology and Oncology, Nanjing Children's Hospital Affiliated with Nanjing Medical University, Nanjing, 'Department of Hematology and Oncology, Shanghai Children's Medical Center Affiliated with Shanghai Jiao Tong University, Shanghai, 'Department of Hematology and Oncology, Soochow Children's Hospital Affiliated with Soochow University, Soochow, dDepartment of Molecular and Genetic Toxicology, Cancer Center of Nanjing Medical University, Nanjing, China
\end{abstract}

\author{
Key Words \\ SNP • FOXO3a $•$ Childhood ALL $・$ miR-223 $・$ Transfection
}

\begin{abstract}
Background/Aims: FOXO3a is an essential tumor suppressor that regulates the mechanisms of tumorigenesis and leukemogenesis. FOXO3a polymorphisms have not been reported previously associated with an increased risk for childhood acute lymphoblastic leukemia (ALL). In this study, the rs4946936 polymorphism located in the 3'UTR of FOXO3a was selected to evaluate its relationship with a risk for ALL in Chinese children. Methods: Questionnaires, SNaPshot genotyping, real-time PCR, cell transfection and dual luciferase reporter assays were used in this study. Results: Parental alcohol consumption and whether the child's house had been painted had significantly different distributions among the cases and controls. In addition, the $\mathrm{C}$ to $\mathrm{T}$ mutation was significantly associated with the risk for ALL. We determined that $\mathrm{FOXO} a$ expression levels in patients with the $\mathrm{CT}$ or TT genotype were significantly higher than those of patients with the CC genotype. The T allele significantly increased the expression levels in luciferase assays and affected the binding affinity of miR-223 to the FOXO3a 3'UTR. Conclusion: Rs4946936 in FOXO3a was highly associated with an increased risk of childhood $\mathrm{ALL}$ in a Chinese population.
\end{abstract}

Y. Wang, L. Zhou and J. Li contributed equally. 


\section{Introduction}

Acute lymphoblastic leukemia (ALL), the most common cancer among children, is a clonal hematological disease in which undifferentiated or poorly differentiated lymphocytes proliferate indefinitely in hematopoietic tissue [1]. Only a small percentage of children exposed to environmental risk factors such as benzene, ionizing radiation or infectious agents suffer from ALL, however, suggesting that genetic variation plays a critical role in disease development.

Recently, genome-wide association studies (GWAS) and candidate gene approaches have been employed to identify susceptibility loci for ALL. The first GWAS performed for childhood ALL identified risk loci on 7p12.2, 10q21.2 and 14q11.2, which mapped to the IKZF1, ARID5B and CEBPE genes, respectively [2]. The genes identified from the GWAS play roles in transcriptional regulation and differentiation of B-cell progenitors. Our previous study as well as studies from other laboratories found that polymorphisms in methylenetetrahydrofolate reductase (MTHFR), a gene critical for DNA methylation and nucleotide synthesis, are associated with a decreased risk for childhood ALL [3].

FOXO3a, a member of the Forkhead family of transcription factors, regulates the cell cycle, cell growth, apoptosis, DNA damage responses and angiogenesis [4], and has previously been implicated as a gene that promotes longevity, and several SNPs (e.g. rs13217795, rs2764264, rs479744, rs2802292 and rs4946936) were found to be associated with increased lifespan in both females and males in Japanese, German, Italian and Han Chinese populations $[5,6]$. Whether genetic variants of this gene contribute to other diseases such as childhood ALL is still unclear.

MicroRNAs (miRNAs) are small, non-coding RNA molecules that range from 19-25 base pairs in length and regulate gene expression through transcriptional repression of target mRNAs [7]. Previous studies have shown that SNPs located in miRNA binding sites within the 3'UTR may affect the binding affinities between a miRNA and its target mRNA, thus affecting the expression level and function of the target gene[8]. Altered binding between a miRNA and its target may also potentially be associated with various cancers. Here, we present a novel association between a polymorphism in the FOXO3a 3'UTR and childhood ALL.

\section{Materials and Methods}

\section{Rs4946936 polymorphism selection}

We designed our study such that SNPs of interest were located in a potential functional region of FOXO3a and had a minor allele frequency (MAF) value greater than $5 \%$ in the Chinese population. Using these criteria, we identified only one SNP (rs4946926) using Pubmed and the Hapmap database.

\section{Study subjects}

This was a hospital-based case-control study that contained 753 patients newly diagnosed with childhood ALL and 1,088 cancer-free controls. Subjects ranged in age from 1 to 18 years old. Study subjects were genetically unrelated from a Han Chinese population between January 2007 and January 2013. The cases were recruited from the Affiliated Nanjing Children's Hospital of Nanjing Medical University (Nanjing, China), the Affiliated Shanghai Children's Medical Center of Shanghai Jiao Tong University (Shanghai, China) and the Affiliated Soochow Children's Hospital of Soochow University (Suzhou, China). Cancer-free control subjects were selected from the same geographic area and showed no evidence of genetic relationship with the cases and the controls had no evidence of malignant neoplasms or hematological diseases.

All patients were diagnosed by Morphology, Immunology, Cytogenetics and Molecular Biology (MICM) according to the established guidelines for diagnosis and treatment of childhood ALL in China (Society of Pediatrics, Chinese Medical Association, 2006). Children with other hematological disorders, a previous history of cancer, radiotherapy or chemotherapy were excluded from this study. All cases and controls were age- $( \pm 5$ years $)$ and gender-matched. A questionnaire was administered in person to study subjects to collect demographic data and environmental exposure information, including the house painting status, 
Wang et al.: Polymorphism rs4946936 Associated with ALL

parental alcohol use and cigarette use status. Before administering the questionnaire, we obtained written informed consent from parents of suitable subjects. Parental drinking status was considered negative if neither of the parents imbibed alcohol. Parental smoking status was defined as negative if neither parents smoked during or after the pregnancy. The house painting status was considered negative if the house was not painted during the pregnancy or after birth. Our study was approved by the Institutional Review Board of the Affiliated Nanjing Children's Hospital of Nanjing Medical University.

\section{Genotyping}

Genomic DNA was extracted from the peripheral blood of every validated subject using the QIAamp DNA Blood Mini Kit (Qiagen, Germany) according to the manufacturer's instructions. The rs4946936 genotypes were partially (342 cases and 485 controls) detected using the polymerase chain reactionrestriction fragment length polymorphism (PCR-RFLP) method. The fragment was amplified using the following primers: FOXO3a forward primer 5'-CAAAATAGCTACTTACCTTTGCAGAT-3'; reverse primer 5 '-CAACAAACGCTAGAAAAGGAGA-3'. The remaining genotyping was done using the SNaPshot genotyping approach (Shanghai Generay Biotech Co., Ltd). Approximately $10 \%$ of samples were randomly selected for repeat analysis for confirmation of the genotype, and the results were $100 \%$ concordant.

\section{Real-time PCR}

Real-time PCR (RT-PCR) was performed to determine whether the $\mathrm{C}$ to $\mathrm{T}$ mutation altered the FOXO3a expression level. We randomly selected 48 ALL patients from each of the three genotypes to assay expression levels by PCR (CC genotype: 25 subjects; CT genotype: 13 subjects; TT genotype: 10 subjects). The primer sequences (Invitrogen Life Technologies Co, CA, USA) were as follows: forward primer 5'-TCACGCACCAATTCTAACGC-3'; reverse primer 5'-CACGGCTTGCTTACTGAAGG-3'. GAPDH was used as an internal control and the forward primer sequence was 5'-GCACCGTCAAGGCTGAGAAC-3'; the reverse primer sequence was 5'-GGATCTCGCTCCTGGAAGATG-3'. The amplicons were electrophoresed on a $2 \%$ agarose gel, stained with ethidium bromide and imaged using an ultraviolet (UV) transilluminator. We quantified the PCR products by measuring the integrated densities for each of the resulting bands using Image J software (version 1.32, NIH).

\section{Cell lines and cell culture}

Jurkat cells (derived from a 14-year-old T-cell lymphoblastic leukemia patient) were a kind gift from Professor Y. Sun (Department of Cell Biology and Medical Genetics, Nanjing Medical University, Nanjing, China). Jurkat cells were maintained in RPMI-1640 medium supplemented with $10 \%$ fetal bovine serum (FBS). HEK 293T cells were obtained from the American Type Culture Collection (ATCC, Manassas VA, USA) and maintained in Dulbecco's modified eagle medium (DMEM) supplemented with 10\% FBS. Cells were cultured at $37^{\circ} \mathrm{C}$ with $95 \%$ air $/ 5 \% \mathrm{CO}_{2}$ in a humidified incubator.

\section{Prediction of miRNA binding to the SNP}

A bioinformatics approach was used to identify miRNAs predicted to bind to the 3'UTR of FOXO3a containing the rs4946936 site, using the PITA Database (http://genie.weizmann.ac.il/pubs/mir07). We found that Hsa-miR-15a and Hsa-miR-223 specifically bind to the sequence surrounding the polymorphism (Fig. 1).

Construction of luciferase-based reporter plasmids

An 840 bp fragment from the FOX03a 3'UTR (from 431 bp upstream of the polymorphism to 408 bp downstream of the site) containing either the $\mathrm{C}$ or $\mathrm{T}$ allele for rs 4946936 was amplified from genomic DNA of HEK 293T cells. The PCR amplicon containing the 3'UTR fragment was subcloned into the pGL3promoterless luciferase-based vector (Promega) between the KpnI and XhoI restriction sites. The sequence of the amplified fragment was verified by DNA sequencing.

\section{Transient transfection}

Electroporation was used for transfection of Jurkat cells. Briefly, cells were centrifuged, separated from the culture media and washed with phosphate-buffered saline (PBS). Cells were then resuspended in RPMI-1640 and transferred into $4 \mathrm{~mm}$ electroporation cuvettes (Bio-Rad, Hercules, CA, USA) in $350 \mu \mathrm{L}$ 
Fig. 1. Potential miRNA binding sites adjacent to the rs4946936 polymorphism in the FOXO3a 3'UTR.

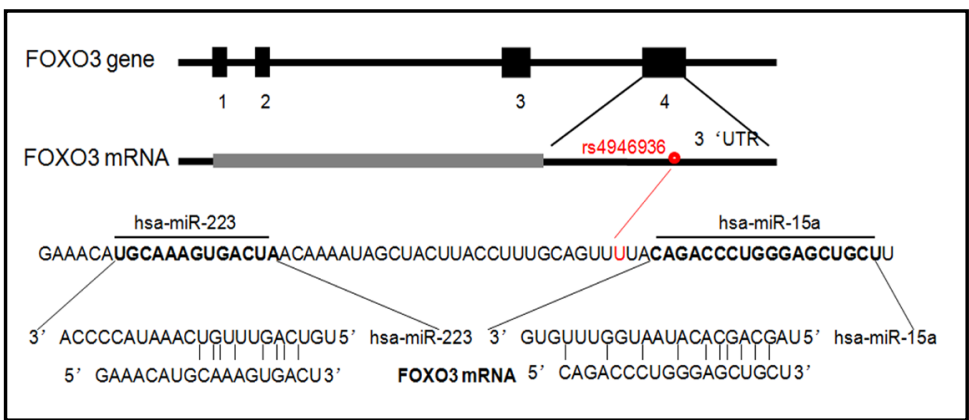

of solution $10^{7}$ cells per cuvette. pGL3-basic plasmid $(10 \mu \mathrm{g})$ with either PGL3 control empty vector, the rs4946936 C allele or the rs4946936 T allele construct (Generay Biotech, Shanghai, China) was added into the solution in conjunction with $20 \mu \mathrm{l}(20 \mu \mathrm{M})$ miRNA (miR-15a or miR-223) mimics or a negative control (GenePharma, Shanghai, China). Additionally, the pRL-SV40 Renilla luciferase vector was co-transfected into cells as an internal control at 1:1000 with the pGL3-vectors described above to reduce experimental variability and normalize detection. We used the Gene Pulser II (Bio-Rad, Hercules, CA, USA) with the following electroporation conditions: $220 \mathrm{~V}$ pulse, $975 \mathrm{uF}$ at room temperature. After electroporation, cells were incubated on ice for 15 minutes and then transferred into $6 \mathrm{~cm}$ plates with $8 \mathrm{~mL}$ medium without antibiotics. For HEK 293T cells, Lipofectamine 2000® (Invitrogen Corp, CA, USA) was used for transfection according to the manufacturer's protocol. Cells were seeded on 24 -well plates with $10^{5}$ cells per well and co-transfected with $2.25 \mu \mathrm{g}$ pGL3-basic vector, $10 \mathrm{ng}$ pRL-SV40 and miR-223 or miR-15a mimics or native controls at $50 \mathrm{nM}$.

\section{Dual-luciferase reporter assay}

Forty-eight hours after transfection, cells were washed with PBS and lysed using Passive Lysis Buffer (Promega, Madison, WI, USA). Firefly and Renilla luciferase activities were determined using the DualGlo Luciferase Reporter Assay System (Promega, Madison, WI, USA) with a Centro LB 960 microplate luminometer (Berthold, Germany), and the relative luciferase activity (a Firefly:Renilla luciferase ratio) was measured according to the manufacturer's instructions. Independent experiments were performed in triplicate for all samples.

\section{Statistical analysis}

Hardy-Weinberg equilibrium was evaluated using a goodness of fit $\chi^{2}$-test to analyze the genotype distribution among control subjects to identify possible selection bias and genotyping errors. An association between the rs 4946936 genotypes and a risk for ALL was evaluated by calculating the odds ratios (ORs) and their $95 \%$ confidence intervals (95\% CIs) using univariate and multivariate logistic regression analysis. To determine the genotype distribution in cases and controls and their association for ALL risk, stratification analysis was performed according to the immunophenotype and risk classification. Differences in expression levels of FOXO3a from the three genotypes (represented by the ratios of the intensity values of FOXO3a and GAPDH) and the difference of the relative luciferase activities between the wildtype and mutant genotypes were evaluated by an independent sample $t$ test. All statistical tests were two-tailed and $P<0.05$ was set as the threshold for statistical significance. Statistical analysis was performed with SPSS 13.0 (SPSS Ltd., Chicago, IL, USA) and SAS software (version 9.1.3; SAS Institute, Cary, NC, USA). Graphs were generated using Graphpad Prism 5.0 (Graphpad Software, Inc., La Jolla, CA, USA).

\section{Results}

Differences in the distribution of the selected demographic variables among controls and ALL are shown in Table 1 . No significant differences in age $(P=0.204)$, sex $(P=0.890)$ or parental smoking status $(P=0.084)$ were identified between the case and control groups. In the case group, there were more parents positive for alcohol consumption $34.5 \%$ versus $21.0 \% ; P<0.0001)$. In addition, significantly more ALL cases than controls had had their 
Table 1. Frequency distributions of selected variables in ALL cases and cancer-free controls
Table 2. Genotype frequencies of the FOXO3a rs4946936 C>T polymorphism among ALL cases and controls and associations

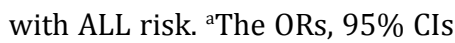
and $P$ value were calculated after adjusting for age, gender, parental smoking, drinking and house painting status

Table 3. Association between the rs4946936 polymorphism and immunophenotype and the risk classification for ALL. ${ }^{\text {aThe ORs, }}$ 95\% CIs and $P$ value were calculated after adjusting for age, gender, parental smoking and drinking status, and house painting status. ${ }^{\text {bTwo }}$ cases were subtypes other than T- or B-ALL

\begin{tabular}{lccccc}
\hline Variables & $\begin{array}{c}\text { Cases } \\
n\end{array}$ & $\begin{array}{c}n=753) \\
\%\end{array}$ & $\begin{array}{c}\text { Controls } \\
n\end{array}$ & $\begin{array}{c}n=1088) \\
\%\end{array}$ & $P$ \\
\hline $\begin{array}{l}\text { Age (years) } \\
\leq 5\end{array}$ & 404 & 53.6 & 551 & 50.6 & 0.204 \\
$>5$ & 349 & 46.4 & 537 & 49.4 & \\
$\begin{array}{l}\text { Gender } \\
\text { Male }\end{array}$ & 462 & 61.3 & 671 & 61.7 & 0.890 \\
Female & 291 & 38.7 & 417 & 38.3 & \\
$\begin{array}{l}\text { Parental smoking status } \\
\text { Negative }\end{array}$ & 314 & 41.7 & 498 & 45.8 & 0.084 \\
$\begin{array}{l}\text { Positive } \\
\text { Parental drinking status }\end{array}$ & 439 & 58.3 & 590 & 54.2 & \\
Negative & 493 & 65.5 & 860 & 79.0 & $<0.0001$ \\
$\begin{array}{l}\text { Positive } \\
\text { House painting status }\end{array}$ & 260 & 34.5 & 228 & 21.0 & \\
Negative & 483 & 64.1 & 803 & 73.8 & $<0.0001$ \\
Positive & 270 & 35.9 & 285 & 26.2 & \\
\hline
\end{tabular}

\begin{tabular}{|c|c|c|c|c|c|c|}
\hline \multirow{2}{*}{ Genotype } & \multicolumn{2}{|c|}{ Cases $(n=753)$} & \multicolumn{2}{|c|}{ Controls $(n=1088)$} & \multirow{2}{*}{$\begin{array}{l}\text { Adjusted OR } \\
(95 \% \mathrm{CI})^{\mathrm{a}}\end{array}$} & \multirow{2}{*}{ Adjusted $P$ Value } \\
\hline & $n$ & $\%$ & $n$ & $\%$ & & \\
\hline $\mathrm{CC}$ & 347 & 46.1 & 593 & 54.5 & 1.00 & 0.002 \\
\hline СТ & 358 & 47.5 & 440 & 40.4 & $1.33(1.10-1.62)$ & \\
\hline TT & 48 & 6.4 & 55 & 5.1 & $1.54(1.01-2.34)$ & \\
\hline $\mathrm{CT}+\mathrm{TT}$ & 406 & 53.9 & 495 & 45.5 & $1.35(1.12-1.63)$ & 0.002 \\
\hline $\mathrm{T}$ allele & 0.301 & & 0.253 & & & 0.001 \\
\hline
\end{tabular}

\begin{tabular}{lcccccc}
\hline Variables & \multicolumn{2}{c}{$\mathrm{CC}$} & \multicolumn{2}{c}{ CT/TT } & Adjusted $P$ Value & Adjusted OR \\
& $n$ & $\%$ & $n$ & $\%$ & & \\
\hline $\begin{array}{l}\text { Immunophenotype } \\
\text { b }\end{array}$ & & & & & & \\
B-ALL & 302 & 46.2 & 352 & 53.8 & 0.002 & $1.37(1.12-1.66)$ \\
T-ALL & 43 & 44.3 & 54 & 55.7 & 0.12 & $1.40(0.92-2.14)$ \\
Risk classification & & & & & & \\
Low-risk ALL & 129 & 42.0 & 178 & 58.0 & 0.0005 & $1.59(1.23-2.07)$ \\
Medium-risk ALL & 71 & 48.0 & 77 & 52.0 & 0.166 & $1.28(0.90-1.80)$ \\
High-risk ALL & 147 & 49.3 & 151 & 50.7 & 0.218 & $1.18(0.91-1.53)$ \\
\hline
\end{tabular}

house painted during the mother's pregnancy or after the child's birth $(35.9 \%$ versus $26.2 \%$; $P<0.0001$ ).

The genotypic distribution of the FOXO3a rs4946936 C>T for cases and controls is presented on Table 2. Hardy-Weinberg equilibrium was observed for genotype distribution in the controls. The genotype distribution was statistically significant between the case and control groups $(P=0.002)$. The T allele was more common in the ALL group $(30.1 \%)$ compared with the control group $(25.3 \%)$ and the difference was statistically significant $(P$ $=0.001$ ). In the univariate logistic regression analysis, the CT genotype frequency in the two groups had an OR of 1.33 and the $95 \%$ CI ranged from 1.10 to 1.62 while the TT genotype had an OR of 1.54 and the $95 \% \mathrm{CI}$ ranged from 1.01 to 2.34 . Results were more significant when the dominant model was applied $(\mathrm{OR}=1.35,95 \% \mathrm{CI}=1.12-1.63, P=0.002)$. The combined CT/TT genotype was associated with an increased risk of childhood ALL.

In a further analysis, we observed an association between the rs 4946936 polymorphism and B-subtype ALL and low risk ALL (Table 3). The combined CT/TT genotype was associated with a significantly increased risk for both B-subtype ALL (OR = 1.37, 95\% CI = $1.12-1.66$, $P=0.002)$ and low risk ALL (OR $=1.59,95 \% \mathrm{CI}=1.23-2.07, P=0.0005)$. No significant association, however, was identified between the genotypes and T-subtype ALL, medium risk or high risk ALL. 
Fig. 2. FOХOЗa expression levels in patients with the CC, CT and TT genotypes. Relative expression levels were calculated by densitometric quantification of the resulting PCR products. * $P=0.009$ (expression was normalized to the CC genotype), ${ }^{* *} P=0.0001$ (expression was normalized to the CC genotype).
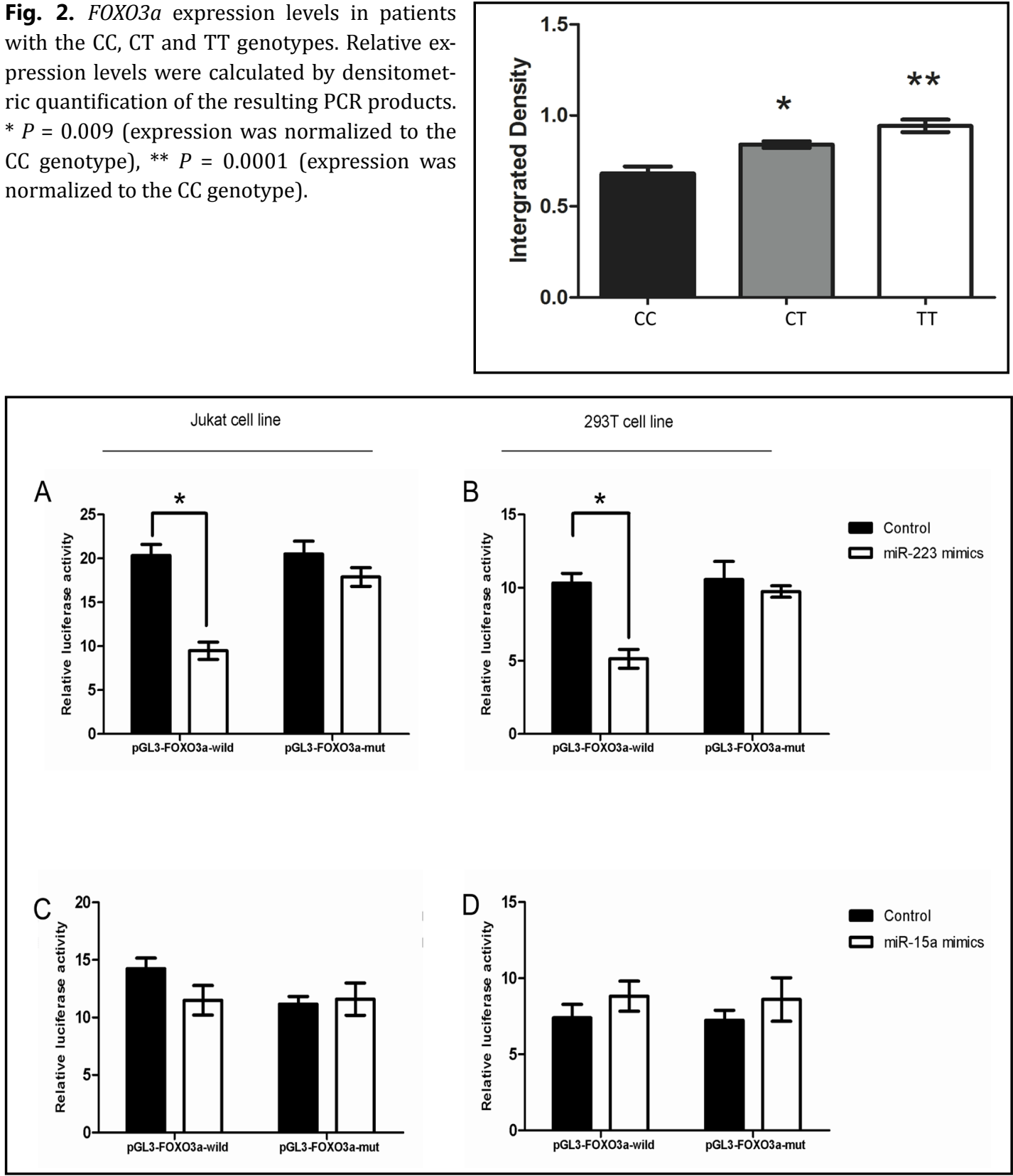

Fig. 3. Relative luciferase activity after co-transfection of miR-223 or miR-15a with FOXO3a polymorphismspecific constructs into Jurkat and HEK 293T cells. The only difference between the two FOXO3a 3'UTR constructs was a $\mathrm{C}$ or T allele at the rs4946936 polymorphism in the 3'UTR. All constructs were co-transfected with miR-223 (A,B) or miR-15a (C,D). pGL3-FOXO3a-mut contains the T allele at rs4946936 site while pGL3-FOXO3a-wild contains the wildtype $C$ allele at the polymorphism. $\left({ }^{*} P<0.05\right)$.

Using RT-PCR, a significant difference in the FOXO3a expression levels of patients carrying CC, CT and TT genotype was observed (Fig. 2). The expression levels of the CT carriers $(P=0.009)$ and the TT carriers $(P=0.0001)$ were higher than those of CC carriers. We also observed a statistically significant difference between the expression levels of the CT and TT carriers $(P=0.028)$.

To determine if the rs4946936 polymorphism affected the potential binding of miRNAs (i.e. miR-15a and miR-223) to the 3'UTR of FOXO3a mRNA, Jurkat and 293T cells were 
transiently transfected with the FOXO3a 3'UTR luciferase construct, containing either the wildtype $\mathrm{C}$ allele or the mutant $\mathrm{T}$ allele. Relative activities were measured with a Dual-Glo Luciferase Reporter Assay System (Fig. 3). Co-transfection of the luciferase vector with the 3'UTR of FOXO3a containing the mutated T allele and Hsa-miR-223 into Jurkat cells significantly altered the luciferase expression level compared with the reporter plasmid containing the wildtype allele. While the wildtype allele construct showed reduced luciferase in the presence of miR-223, luciferase levels in the mutant $\mathrm{T}$ allele construct in the presence of miR-223 did not appreciably change. Co-transfection of Hsa-miR-15a did not alter expression levels in wild or mutated FOXO3a allele constructs. The same results were observed in HEK 293T cells. Thus, we conclude that the rs4946936 polymorphism alters the binding affinity of miR-223, but not miR-15a, with the 3'UTR of FOXO3a mRNA.

\section{Discussion}

After administering the questionnaire and analyzing the results, we identified parental alcohol consumption and whether the house had been painted during the child's life as risk factors for childhood ALL. Parental alcohol consumption and painting of the house pose potentially hazardous environmental exposures for children, and increase the risk for ALL. These findings lend valuable insight into methods to lower the risk for childhood ALL.

FOX03a belongs to the Forkhead family of transcription factors, which has three other members: FOXO1, FOXO4 and FOXO6. FOXO family members function as tumor suppressors but also play vital roles in a diverse array of cellular processes such as differentiation, apoptosis, cell cycle arrest, metabolism and responses to cellular stress [9]. FOXO3a is $124.94 \mathrm{~kb}$ in length and located on chromosome 6q21. FOXO3a activity is regulated through post-translational modifications such as methylation, acetylation and ubiquitination [10]. Recently, reports examining the role of FOXO proteins in leukemia have shown that FOXO3a is constitutively inactive in acute myeloid leukemia (AML) blast cells due to aberrant cytoplasmic localization [11]. High expression levels of FOXO3a mRNA and phosphorylation of FOXO3a were found to be adverse prognostic factors for AML and were associated with increased proliferation, resistance to therapeutics and shorter relapse-free intervals $[12,13]$. In addition, increased expression and nuclear accumulation of FOXO3a causes a feedback loop that enhances PI3K/AKT activity, which ultimately drives tumor cell survival and drug resistance in chronic myelogenous leukemia cells [14]. These reports indicate that maintenance of proper FOXO3a levels is critical in preventing leukemogenesis. Here, we hypothesize that functional genetic changes in FOXO3a-in the form of polymorphisms-alter the activity of the transcription factor and increase susceptibility for childhood ALL.

In this study, we identified the rs4946936 polymorphism located in the 3'UTR of FOX03a to investigate its association with childhood ALL in a Han Chinese population. This polymorphism has never been examined in relationship with particular diseases. We found that the $\mathrm{C}$ to $\mathrm{T}$ mutation of rs4946936 significantly increased the risk of ALL. Moreover, the mutation was associated with a higher risk of B-phenotype ALL and low-risk ALL in a stratification analysis. The expression levels of FOXO3a mRNA were significantly higher in the patients carrying either the CT or TT genotype, as compared to patients carrying the CC genotype. Furthermore, a dual-luciferase reporter assay suggested that the $\mathrm{T}$ allele significantly affected the binding affinity of miR-223 to the FOXO3a 3'UTR, which would ultimately affect the FOXO3a transcript stability.

Due to experimental design limitations, we only selected two miRNAs in close proximity to the polymorphism on the mRNA. Other as of yet unidentified miRNAs interacting with the rs4946936 polymorphism may also regulate FOXO3a gene expression and function.

In conclusion, parental alcohol consumption and house painting during the child's life were identified as environmental risk factors for childhood ALL. Our study is the first to demonstrate that the rs4946936 polymorphism in the 3'UTR of FOXO3a is highly associated with an increased risk for childhood ALL in a Han Chinese population. In addition, further 
functional characterization of the polymorphism's effect on miRNA binding and FOXO3a stability may elucidate the underlying mechanisms for ALL etiology as well as potential therapeutic target for treatment.

\section{Disclosure Statement}

None declared.

\section{Acknowledgments}

This research was supported in part by the National Natural Science Foundation of China (81070436) and the Natural Science Foundation of Jiangsu Province (BK2011775).

\section{References}

1 Pui CH: Acute lymphoblastic leukemia in children. Curr Opin Oncol 2000;12:3-12.

-2 Papaemmanuil E, Hosking FJ, Vijayakrishnan J, Price A, Olver B, Sheridan E, Kinsey SE, Lightfoot T, Roman E, Irving JA, Allan JM, Tomlinson IP, Taylor M, Greaves M, Houlston RS: Loci on 7p12.2, 10q21.2 and 14q11.2 are associated with risk of childhood acute lymphoblastic leukemia. Nat Genet 2009;41:1006-1010.

3 Tong N, Fang Y, Li J, Wang M, Lu Q, Wang S, Tian Y, Rong L, Sun J, Xu J, Zhang Z: Methylenetetrahydrofolate reductase polymorphisms, serum methylenetetrahydrofolate reductase levels, and risk of childhood acute lymphoblastic leukemia in a chinese population. Cancer Sci 2010;101:782-786.

-4 Tsai WB, Chung YM, Takahashi Y, Xu Z, Hu MC: Functional interaction between foxo3a and atm regulates DNA damage response. Nat Cell Biol 2008;10:460-467.

-5 Li Y, Wang WJ, Cao H, Lu J, Wu C, Hu FY, Guo J, Zhao L, Yang F, Zhang YX, Li W, Zheng GY, Cui H, Chen X, Zhu Z, He H, Dong B, Mo X, Zeng Y, Tian XL: Genetic association of foxo1a and foxo3a with longevity trait in han chinese populations. Hum Mol Genet 2009;18:4897-4904.

6 Anselmi CV, Malovini A, Roncarati R, Novelli V, Villa F, Condorelli G, Bellazzi R, Puca AA: Association of the foxo3a locus with extreme longevity in a southern italian centenarian study. Rejuvenation Res 2009;12:95104.

7 Yu Z, Li Z, Jolicoeur N, Zhang L, Fortin Y, Wang E, Wu M, Shen SH: Aberrant allele frequencies of the snps located in microrna target sites are potentially associated with human cancers. Nucleic Acids Res 2007;35:4535-4541.

-8 Naccarati A, Pardini B, Stefano L, Landi D, Slyskova J, Novotny J, Levy M, Polakova V, Lipska L, Vodicka P: Polymorphisms in mirna-binding sites of nucleotide excision repair genes and colorectal cancer risk. Carcinogenesis 2012;33:1346-1351.

-9 Downing JR: A new foxo pathway required for leukemogenesis. Cell 2011;146:669-670.

10 Yang JY, Hung MC: Deciphering the role of forkhead transcription factors in cancer therapy. Curr Drug Targets 2011;12:1284-1290.

11 Chapuis N, Park S, Leotoing L, Tamburini J, Verdier F, Bardet V, Green AS, Willems L, Agou F, Ifrah N, Dreyfus F, Bismuth G, Baud V, Lacombe C, Mayeux P, Bouscary D: Ikappab kinase overcomes pi3k/akt and erk/mapk to control foxo3a activity in acute myeloid leukemia. Blood 2010;116:4240-4250.

-12 Santamaria CM, Chillon MC, Garcia-Sanz R, Perez C, Caballero MD, Ramos F, de Coca AG, Alonso JM, Giraldo P, Bernal T, Queizan JA, Rodriguez JN, Fernandez-Abellan P, Barez A, Penarrubia MJ, Vidriales MB, Balanzategui A, Sarasquete ME, Alcoceba M, Diaz-Mediavilla J, San Miguel JF, Gonzalez M: High foxo3a expression is associated with a poorer prognosis in aml with normal cytogenetics. Leuk Res 2009;33:17061709.

13 Kornblau SM, Singh N, Qiu Y, Chen W, Zhang N, Coombes KR: Highly phosphorylated foxo3a is an adverse prognostic factor in acute myeloid leukemia. Clin Cancer Res 2010;16:1865-1874.

14 Hui RC, Gomes AR, Constantinidou D, Costa JR, Karadedou CT, Fernandez de Mattos S, Wymann MP, Brosens JJ, Schulze A, Lam EW: The forkhead transcription factor foxo3a increases phosphoinositide-3 kinase/akt activity in drug-resistant leukemic cells through induction of pik3ca expression. Mol Cell Biol 2008;28:5886-5898. 